\title{
Palliative care and public health an issue to be held
}

\begin{abstract}
Palliative Care ( $\mathrm{PC}$ ) is underdeveloped in most parts of the world. $\mathrm{PC}$ is based on knowledge inherent to the various specialties, possibilities of clinical and therapeutic intervention in the various areas of medical science knowledge and specific knowledge. The practice of PC should be adapted to each country or region according to relevant aspects such as availability of material and human resources, type of existing health planning, cultural and social aspects of the population served. PC has received very little attention in the field of public health. The probable reasons are that public health's primary focuses are to prevent illness and premature deaths, and public health professionals may also believe that endof-life issues are a health system problem rather than a priority to be addressed through population health efforts. With the rapidly aging world population and the associated increase of multiple "noncommunicable" diseases, the need for palliative care will increase dramatically over the next 50 years. Although palliative care services may start in one or more health care organizations that will become centers of PC excellence, it is always important to keep in mind the vision that the process to implement palliative care within a country is striving to integrate palliative care into all levels of the society from the community level upward and from the palliative care expert in the health care system downward. Given this, the creation of Palliative Care teams should be among the priorities of managers, due to the demographic transition and the high demand for palliative care due to the growth of morbidities secondary to chronic degenerative diseases
\end{abstract}

Keywords: palliative care, public health, hospices, hospice care
Volume 5 Issue I - 2020

\author{
Bruno Bastos Godoi, ${ }^{1,4}$ Luiza Vilas Boas \\ Freitas,' Alice Assis Chaves,' Barbara \\ Machado Alfradique,' Isabella Ferreira \\ Brugiolo,' Maria Fernanda Nobre Leão, ${ }^{2}$ \\ Giovana Amaral Cordeiro, ${ }^{3,4}$ Fabiana Souza \\ Máximo Pereira ${ }^{3,4}$ \\ 'Student, Faculty of Medicine, Federal University of \\ Jequitinhonha and Mucuri Valleys, Brazil \\ 2Medical Clinic Resident, Faculty of Medicine, Federal University \\ of Jequitinhonha and Mucuri Valleys, Brazil \\ ${ }^{3}$ Professor, Faculty of Medicine, Federal University of \\ Jequitinhonha and Mucuri Valleys, Brazil \\ ${ }^{4}$ Palliative Care Team, Santa Casa de Caridade, Brazil
}

Correspondence: Bruno Bastos Godoi, Faculty of Medicine, Palliative Care Team, Federal University of Jequitinhonha's 'Valley and Mucuri, Santa Casa de Caridade, Diamantina/MG, Brazil, Tel +55(3I)9868625II, Email bastosgodoi@gmail.com

Received: December 13, 2019 | Published: February 06, 2020

\section{Introduction}

The need for palliative care (PC) has never been greater and is increasing at a rapid pace due to the aging world population and rising cancer and other noncommunicable diseases. ${ }^{1,2}$ Despite this need, $\mathrm{PC}$ is underdeveloped in most parts of the world. PC is expanding in the developed world, despite the myths and misunderstanding about its nature and purpose, but it is just beginning to be available in the developing world, where it is most needed..$^{3-8}$

Another concept to overcome is the patient who is "out of therapeutic possibilities". There is always a therapy to be recommended for a patient. In the advanced stage of a disease and with little chance of cure, physical symptoms are factors of discomfort. For these there are procedures, medicines and approaches that can provide physical well-being until the end of life. This therapy cannot be denied to the patient. ${ }^{9-11}$

The path of adequate information, the formation of competent professional teams, the reaffirmation of the principles of PC and the demonstration of positive results of this treatment modality, is the best way to overcome remaining barriers to the implementation of a PC policy. ${ }^{9-11}$

PC is based on knowledge inherent to the various specialties, possibilities of clinical and therapeutic intervention in the various areas of medical science knowledge and specific knowledge. World Health Organization (WHO) in 1986 published principles governing the work of the multidisciplinary PC team. These principles were reaffirmed in their review in 2002: promote pain relief and unpleasant symptoms; not accelerate or prorogate death; integrate psychological and spiritual aspects in patient care; offer support systems that enable the patient to live as actively as possible until the moment of death; provide support system to assist family members during the patient's illness and to cope with grief; multiprofessional approach to addressing the needs of patients and their families, including bereavement followup; improve the quality of life and positively influence the course of the disease; should be started as early as possible, along with other life-prolonging measures such as chemotherapy and radiotherapy, and include all necessary investigations to better understand and control stressful clinical situations. ${ }^{10,12-15}$

\section{Palliative care and public health}

The practice of PC should be adapted to each country or region according to relevant aspects such as availability of material and human resources, type of existing health planning, cultural and social aspects of the population served. This should be done according to the scope of PC presented in Figure 1. ${ }^{10,14,16}$

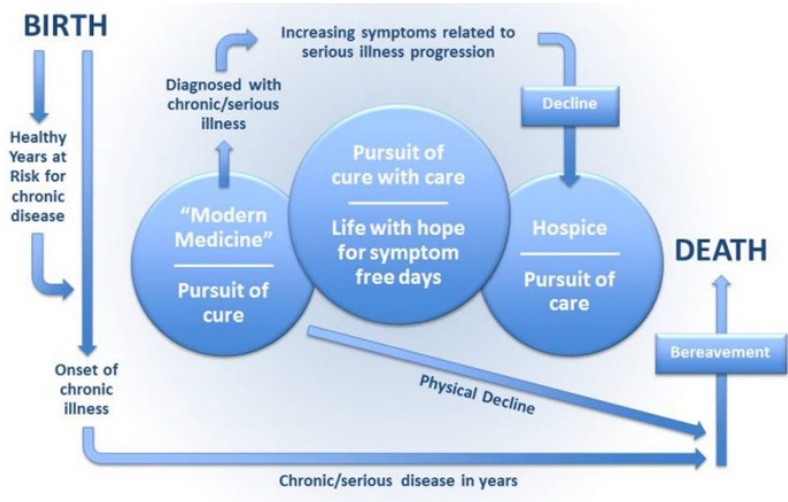

Figure I Scope of palliative care. ${ }^{9}$ 
When talking about active, progressive and life-threatening disease, it means that PC can and should be indicated in the presence of chronic diseases in different stages of evolution. There is the possibility that death by natural evolution of a process of falling ill can be postponed for years. It is not possible to apply the principles of palliative care only in the case when there is a sudden death due to illness, accident or violence. ${ }^{17-19}$

The increased survival has also generated a considerable number of Intensive Care Unit (ICU) patients and, consequently, a greater experience of the health professional in the terminality process. Thus, in order to individualize the care provided and organize care in highly complex environments, palliative care is adopted, which uses advanced communication skills in an attempt to alleviate pain, psychological and spiritual suffering. ${ }^{16,20}$

The use of care protocols in the care of patients under the final conditions of life is extremely important, since it makes health care systematized.

\section{Is PC a public health issue?}

PC has received very little attention in the field of public health worldwide. The reasons are that the primary focus of public health is to prevent any kind of disease or premature death. ${ }^{9-11,13,14,20,21}$

"End-of-life issues have a huge impact on the dying person's family". ${ }^{17}$ We estimate that in 2030 there will be more than 74 million deaths per year and the majority of this deaths linked to chronic diseases..$^{17,20,21}$

Globally we have more than 400,000 PC workers, over 1.2 million volunteers, and over 9 million people acting as palliative caregivers. This means that over 10.5 million people are involved with delivering hospice and PC every year. ${ }^{20}$

\section{Public health and palliative care approach}

WHO has formulated some approaches that can facilitate the PC at public health in order to help more patients who need this kind of care: ${ }^{11,16,20}$

i. Ensure health system policies to integrate PC services at all levels of care

ii. Ensure health system policies to expand human resources, include education, and health care professional training

iii. Ensure health system policies to turn available medicines for the symptoms management, as pain or psychological distress

iv. Ensure health system policies to provide researches in PC in order to improve patients' care

\section{The need for palliative care}

Globally, there is a significant need for PC and its development. At least $60 \%$ of people dying will have a prolonged illness and dying older, necessitating from a service of PC. ${ }^{11,13,16,20}$

With the rapidly aging world population (demographic transition) and the associated increase of multiple "noncommunicable" diseases, the need for palliative care will increase dramatically over the next 50 years: ${ }^{11,13,16,20}$
1. "In 2025 there will be over 1,200 million people of 60 years or older; in 2050 the number will increase over to 2,000 million". ${ }^{21}$

2. "The incidence of cancer will more than double to an estimated incidence of 24 million new cancers per year by 2050 ". ${ }^{21}$

3. "If preventative measures don't work, annual mortality from AIDS will increase to four million in 2015 and six million by 2030". ${ }^{21}$

\section{Palliative care for all}

It is relevant to say that the process of implementing an excellence PC center is needed to be achieved through integration into all levels of the society from the community level upward and from the palliative care expert in the health care system downward. Despite this, it is imperative to develop a PC system linked to the existing health care system and social support network. "It will be critical for all palliative care experts to spend $40 \%-50 \%$ of their time educating and supporting other health care professionals and community support systems" (Figure 2). ${ }^{10-13,16,17,22-25}$

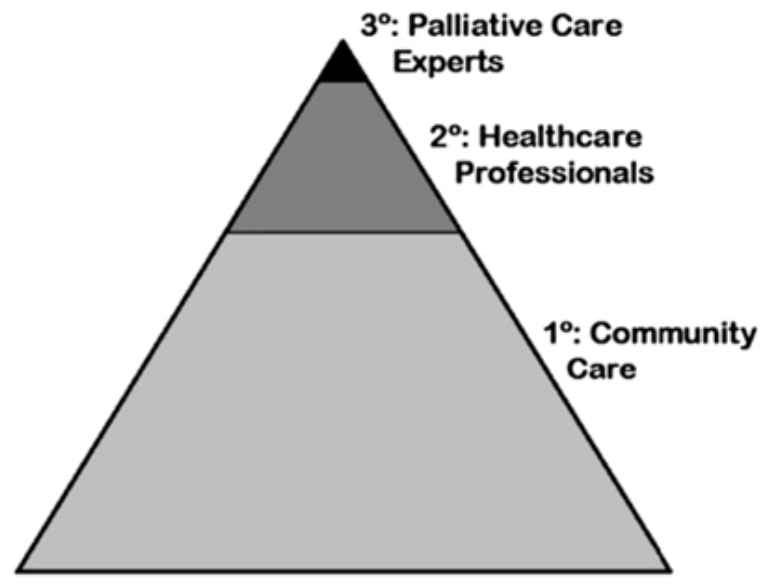

Figure 2 Palliative care for all. ${ }^{16}$

\section{Conclusion}

Palliative Care is an issue that should be solved and delivered for patients who need this kind of assistance. There are several of challenges for effective Palliative Care, such as: a lack of understanding about palliative care in the community; limited funding and underresourced status; stringent national policies related to increasing availability of some pain control drugs necessary for palliative care, and lack of trained human resources.

Given this, the creation of Palliative Care teams should be among the priorities of managers, due to the demographic transition and the high demand for palliative care caused by the growth of morbidities secondary to chronic degenerative diseases.

\section{Funding}

None.

\section{Acknowledgments}

None. 


\section{Conflicts of interest}

The authors declare no conflict of interest.

\section{References}

1. Murray CJL, Lopez AD. Alternative projections of mortality and disability by cause 1990-2020: global burden of disease study. Lancet. 1997;24;349(9064):1498-1504

2. Lopez AD, Murray CCJL. The global burden of disease, 1990-2020 Nature Medicine. 1998;(4):1241-1243.

3. Tavares de Carvalho R, Afonseca Parsons H. Manual de Cuidados Paliativos ANCP Ampliado e atualizado. Acad Nac Cuid Paliativos. 2013;517-530.

4. Gómez-Batiste X, Lasmarías C, Amblàs J, et al. Chair ICO/UVICUCC of palliative care at the University of Vic - Central University of Catalonia: an innovative multidisciplinary model of education, research and knowledge transfer. BMJ Support Palliat Care. 2018;9(4):e33.

5. Moorhouse P, Mallery LH. Palliative and therapeutic harmonization: a model for appropriate decision-making in frail older adults. $\mathrm{J} \mathrm{Am}$ Geriatr Soc. 2012;60(12):2326-2332.

6. Connor SR, Cecilia SBM, Baxter S, Hospice palliative care association. In: Samira Beckwith CK \& Hospice H, editors. Global atlas of palliative care at the end of life. 2014

7. Coelho CBT, Yankaskas JR. New concepts in palliative care in the intensive care unit. Rev Bras Ter Intensiva. 2017;29(2):222-30.

8. Bergenholtz H, Jarlbaek L, Hølge-Hazelton B. Generalist palliative care in hospital - Cultural and organisational interactions. Results of a mixedmethods study. Palliat Med. 2015;30(6):558-566.

9. https://www.publichealthnotes.com/palliative-care-necessitychallenges-and-actions-to-be-taken/

10. Beng TS, Guan NC, Jane LE, et al. Health care interactional suffering in palliative care. Am J Hosp Palliat Med. 2014;31(3):307-314.

11. De Lima L, Pastrana T. Opportunities for palliative care in public health. Annu Rev Public Health. 2016:18;37(1):357-374.

12. Sallnow L, Paul S. Understanding community engagement in endof-life care: developing conceptual clarity. Critical Public Health. $2015 ;(25): 231-238$
13. Whitelaw S, Clark D. Palliative care and public health: an asymmetrical relationship?. Palliative Care. 2019(12).

14. Ghosh A, Dzeng E, Cheng MJ. Interaction of palliative care and primary care. Clinics in Geriatric Medicine. 2015;(31):207-218.

15. Robinson MT, Holloway RG. Palliative care in neurology. Mayo Clinic Proceedings. 2017;92(10):1592-601.

16. Stjernswärd J, Foley KM, Ferris FD. The public health strategy for palliative care. J Pain Symptom Manage. 2007;33(5):486-493.

17. Molidor S, Overbaugh KJ, James D, et al. Palliative care and stroke: an integrative review of the literature. J Hosp Palliat Nurs. 2018;20(4):358367.

18. Scaccabarozzi G, Limonta F, Amodio E. Hospital, local palliative care network and public health: how do they involve terminally ill patients? Eur J Public Health. 2017;27(1):25-30.

19. Archibald D, Patterson R, Haraldsdottir E, et al. Mapping the progress and impacts of public health approaches to palliative care: a scoping review protocol. BMJ open. 2016(6):e012058.

20. Dzingina MD, Higginson IJ. Public health and palliative care in 2015. Clinics in Geriatric Medicine. 2015;31(2):253-263.

21. Callaway MV, Connor SR, Foley KM. World health organization public health model: a roadmap for palliative care development. $J$ Pain Symptom Manage. 2018;55(2):S6-13.

22. https://www.un.org/en/development/desa/population/events/other/34 index.asp

23. https://www.un.org/en/development/desa/population/index.asp

24. Sallnow L, Richardson H, Murray SA, et al. The impact of a new public health approach to end-of-life care: A systematic review. Palliative Medicine. 2016;30(3):200-211.

25. Martins Pereira S, Araújo J, Hernández-Marrero P. Towards a public health approach for palliative care: An action-research study focused on engaging a local community and educating teenagers. BMC Palliat Care. 2018;17(1) 